\title{
Neuroimaging findings of cerebral syphilitic gumma
}

\author{
CAO LI ${ }^{1}$, SHI-JIE WANG ${ }^{2}$, GUANG-CAI TANG ${ }^{1}$, LUO-TONG LIU ${ }^{3}$ and GUANG-XIANG CHEN ${ }^{1}$ \\ Departments of ${ }^{1}$ Radiology, ${ }^{2}$ Ultrasonography and ${ }^{3}$ Neurosurgery, \\ The Affiliated Hospital of Southwest Medical University, Luzhou, Sichuan 646000, P.R. China
}

Received November 22, 2018; Accepted June 13, 2019

DOI: $10.3892 /$ etm.2019.8089

\begin{abstract}
Cerebral syphilitic gumma is a rarely reported disease of the central nervous system. Magnetic resonance imaging (MRI) is an important diagnostic method for syphilitic gumma. The present study aimed to describe and characterize neuroimaging results from 6 patients with pathologically diagnosed cerebral syphilitic gumma. The 6 patients (age, 32-61 years) underwent brain CT and MRI, with 1 patient also undergoing whole-body 2-deoxy-2-(fluorine-18)fluoro-D-glucose-positron emission tomography/CT (18F-FDG PET/CT). Non-enhanced CT, conventional T1 weighted imaging (T1WI) and T2WI, diffusion weighted imaging (DWI) and gadolinium-enhanced T1WI images were acquired for all patients. The CT and MRI scans were retrospectively reviewed by two experienced radiologists for consensus on the location, number, size, T1WI, T2WI and DWI signal intensity characteristics, extent of vasogenic oedema, and enhancement patterns. In total, the 6 patients exhibited 10 lesions, nine of which were located in the cerebral hemisphere, primarily in the grey matter. The remaining lesion was located in the fourth ventricle, leading to mild-to-moderate hydrocephalus. The diameters of the identified 10 lesions ranged from 0.9-6.5 cm, with a mean diameter of $3.9 \mathrm{~cm}$. The main feature observed in CT was low density and in MRI the features were T1WI and DWI hypointensity and T2WI hyperintensity. A single case exhibited syphilis gumma with massive haemorrhage. Ring-like or strip-like signs $(n=5)$, accompanied by the dural tail sign $(n=2)$ and homogeneous enhancement $(n=1)$, were noted on T1WI with gadolinium. The 18F-FDG PET/CT performed in one patient of a cerebral syphilis gumma revealed low uptake and metabolism. The present study indicated that
\end{abstract}

Correspondence to: Professor Guang-Xiang Chen, Department of Radiology, The Affiliated Hospital of Southwest Medical University, 23 Taiping Street, Luzhou, Sichuan 646000, P.R. China

E-mail: cgx231y2002@163.com

Abbreviations: HIV, human immunodeficiency virus; VE, vasogenic oedema; MRS, magnetic resonance spectroscopy; PWI, perfusion weighted imaging

Key words: cerebral syphilitic gumma, magnetic resonance imaging, 2-deoxy-2-(fluorine-18)fluoro-D-glucose, positron emission tomography/computerized tomography, neuroimaging gadolinium-enhanced MRI combined with 18F-FDG PET/CT and laboratory examinations are helpful in distinguishing cerebral syphilitic gumma from brain tumors and infectious diseases, therefore avoiding unnecessary surgery.

\section{Introduction}

Syphilis is a bacterial infection caused by the Treponema pallidum subspecies pallidum. The World Health Organization estimated that 5.6 million new cases of syphilis occurred among those aged 15-49 years worldwide in 2012, with a global incidence rate of 1.5 cases per 1,000 people, despite the availability of effective treatments $(1,2)$. The estimated 18 million prevalent cases of syphilis in 2012 translates to a global prevalence of $0.5 \%$ among females and $0.5 \%$ among males aged $15-49$ years, with the highest prevalence in the WHO African Region (3). In China, according to an epidemiological study performed on syphilis cases reported from 31 provinces, autonomous regions, and municipalities between 2000 and 2013, the reported syphilis incidence increased yearly from 6.43 per 100,000 person-years in 2000 to 32.86 per 100,000 person-years in 2013 , with an average annual growth rate of $13.37 \%$ (4).

If untreated, the disease lasts many years and has several stages. Early syphilis consists of primary syphilis, secondary syphilis and early latent syphilis, while late syphilis consists of late latent syphilis and tertiary syphilis (neurosyphilis, cardiosyphilis and gumma). Primary syphilis classically presents as a painless chancre at the site of inoculation after a mean incubation period of 21 days (range: 9-90 days). The primary lesion begins as a raised papule and ulcerates before healing within 3 to 10 weeks, with or without treatment (1). The primary chancre may be ignored by patients. If untreated, the disease progresses to the secondary stage, 4 to 8 weeks after the appearance of the primary lesion, which is characterized by generalized mucocutaneous lesions affecting both skin and mucous membranes. The symptoms and signs of secondary syphilis spontaneously resolve, even without treatment, and if left untreated, the patient enters the latent stage, which is often categorized in two phases: Early latent syphilis (infection for less than two years) and late latent syphilis (infection for two years or more). Patients with unknown duration of infection should be treated for late latent syphilis. If left untreated, most patients will remain in the latent stage. Approximately $25 \%$ will develop the late clinical sequelae of tertiary syphilis (5), which can affect any organ system up to 30 years or more after infection. 
Cerebral syphilitic gumma is a rare disease of the central nervous system that is an unusual type of tertiary syphilis. Due to the lack of knowledge regarding the imaging of cerebral syphilitic gumma, pre-operative misdiagnosis often occurs, with glioma being the most commonly misdiagnosed (6-9). Treatment with penicillin G can treat most gummas and only a few patients with intracranial hypertension crisis or penicillin-resistance require surgical resection $(7,10)$. Therefore, a definitive diagnosis of syphilitic gumma may help clinicians choose an appropriate treatment strategy and reduce the risk of unnecessary surgery. There have been limited case reports describing the neuroimaging features of intracranial syphilitic gumma (6-9). Magnetic resonance imaging (MRI) of a cerebral gumma was first described by Agrons et al (11) in 1991. The purpose of the present study was to characterize the neuroimaging features of 6 cases of syphilitic gumma. In the present study, a retrospective analysis using medical records and neuroimaging data was performed to evaluate the neuroimaging features of syphilitic gumma, with an emphasis on MRI.

\section{Patients and methods}

Patients. From August 2012 to July 2016, 6 consecutive patient records with histologically proven syphilitic gumma were reviewed after receiving approval from the institutional review board at The Affiliated Hospital of Southwest Medical University (Luzhou, China). The 6 patients included 2 females and 4 males, ranging in age from 32 to 61 years, with a mean age of 44.3 years. All patients underwent CT, MRI or positron emission tomography (PET)/CT at The Affiliated Hospital of Southwest Medical University. Five patients with a single lesion underwent complete surgical resection. One patient with multiple lesions underwent resection of the largest lesion on the left parietal lobe and the remaining four lesions were not surgically removed.

$C T$ and MRI examination. All the patients were scanned with a 3.0-T scanner (Koninklijke Philips, N.V.) using an eight-channel SENSE head coil (SENSE acceleration factor of 8). The following MRI sequences were included for the brain MRI: Axial T1-weighted imaging [T1WI; repetition time (TR), 2,000 msec; echo time (TE), $20 \mathrm{msec}$, T2WI (TR, $3,000 \mathrm{msec}$; TE, $80 \mathrm{msec}$ ), fluid-attenuated inversion recovery (TR, 11,000 msec; TE, $125 \mathrm{msec}$ ), diffusion weighted imaging (DWI; TR, 4,000 msec; TE, 64 msec; b-value, 1,000). Axial, sagittal and coronal gadolinium-enhanced $(0.1 \mathrm{mmol} / \mathrm{kg})$ T1WI were acquired. All CT images were obtained using a 4-detector CT scanner (LightSpeed; GE Healthcare) with a $5 \mathrm{~mm}$ section thickness, $120 \mathrm{kV}$ and $250 \mathrm{~mA}$.

Due to multiple brain lesions and the suspicion of brain metastases before operation, 18F-FDG PET/CT was performed in only one patient.

Imaging analysis. Two experienced radiologists working in the Affiliated Hospital of Southwest Medical University (Luzhou, China) retrospectively reviewed the CT and MRI scans by consensus for the location, size, density, $\mathrm{T} 1$ and $\mathrm{T} 2$ signal-intensity characteristics, extent of vasogenic oedema (VE) and enhancement patterns. VE was graded using the following scale: 0 , No oedema and an absence of increased T2 signal surrounding the gumma; I, mild oedema with the width of the oedema $<2 \mathrm{~cm}$; II, moderate oedema with the width of the oedema $>2 \mathrm{~cm}$ and the range being $<50 \%$ of the ipsilateral cerebral hemisphere; III, severe oedema where the range of the oedema was $>50 \%$ of the ipsilateral cerebral hemisphere.

Histopathology. All tissues came from surgically resected specimens. First, all the specimens were fixed at room temperature for $24 \mathrm{~h}$ with $4 \%$ neutral formaldehyde solution, then the tissues were embedded in paraffin, and tissues were sectioned $(4 \mu \mathrm{m})$. Finally, hematoxylin-eosin (HE) staining at room temperature for $55 \mathrm{~min}$ and a light microscopic examination was performed (magnification, x200 or x400).

\section{Results}

Patient clinical features. The present study included 6 patients with a confirmed diagnosis of syphilitic gumma. All 6 patients expressed positive results when tested with the Treponema pallidum antibody and 5 patients expressed positive results in the treponema pallidum particle agglutination test. Out of the 6 patients, 4 demonstrated positive results in the serological rapid plasma reagin test. The Treponema pallidum spirochete was found in pathological tissues using a Warthin-Starry silver stain in 2 of the cases. The human immunodeficiency virus (HIV) test was negative in all 6 patients. All the above tests were completed between August 2012 and July 2016. A single patient died a week after the operation and the other 5 patients received post-operative penicillin treatment, as penicillin 18 million U/day intravenously for a period of 2 weeks. Patient clinical features are summarized in Tables I and II.

The imaging features. The 6 patients exhibited a total of 10 lesions, nine of these lesions were located in the superficial region of the cerebral hemisphere (Figs. 1-4), which involved both grey and white matter. In particular, one lesion was located in the fourth ventricle, leading to mild hydrocephalus (Fig. 5A-E). A single patient had multiple lesions, including five lesions located in the left parietal-occipital lobe and temporal lobe (Fig. 3A). The 10 lesions ranged in size from $0.9-6.5 \mathrm{~cm}$ in diameter, with a mean diameter of $3.9 \mathrm{~cm}$.

The CT and MRI features are summarized in Table III. Four patients were hypointense in T1WI and hyperintense in T2WI. Furthermore, a single patient exhibited hyperintensity under DWI. Furthermore, under T1WI and T2WI, 2 patients exhibited mixed signal intensity with hypointensity, hyperintensity and isointensity, while non-enhanced CT scans revealed haemorrhage and focal haemorrhage in the right frontal lobe and left frontal lobe, respectively (Figs. 2A, B, 4A, B). All 6 patients had grade I-III vasogenic oedema surrounding the masses, and a single case was accompanied by a midline structure shift (Fig. 4A-E). The results of contrast-enhanced MRI demonstrated masses with mild $(n=2)$ or marked $(n=3)$ ring-like or strip-like enhancements in the cerebral hemisphere (Figs. 1D, 2D, 3D, E and 4D). The mass in the fourth ventricle exhibited marked homogeneous enhancement $(n=1)$ (Fig. 5D), which led to mild-to-moderate hydrocephalus. An enhanced dura mater adjacent to the masses was observed in 
Table I. Patient clinical characteristics.

\begin{tabular}{lccccccc}
\hline Case & Sex & Age (years) & RPR & TPPA & Anti-TP & HIV & Treponema pallidum spirochete \\
\hline 1 & M & 56 & $(+)$ & $(+)$ & $(+)$ & $(-)$ & No \\
2 & M & 38 & $(+)$ & $(+)$ & $(+)$ & $(-)$ & Yes \\
3 & M & 61 & $(+)$ & $(+)$ & $(+)$ & $(-)$ & No \\
4 & M & 50 & $(-)$ & $(-)$ & $(+)$ & $(-)$ & Yes \\
5 & F & 40 & $(+)$ & $(+)$ & $(+)$ & $(-)$ & No \\
6 & F & 32 & $(-)$ & $(+)$ & $(+)$ & $(-)$ & \\
\hline
\end{tabular}

M, male; F, female; RPR, rapid plasma regain; TPPA, Treponema pallidum particle agglutination; Anti-TP, Treponema pallidum antibody; HIV, human immunodeficiency virus.

Table II. Patient clinical features.

\begin{tabular}{|c|c|c|c|c|}
\hline Case & Number of lesion(s) & Symptoms and durations & Treatment & Follow-up \\
\hline 1 & Single lesion & $\begin{array}{l}\text { Headache for } \sim 6 \text { months, and } \\
\text { vomiting for } \sim 1 \text { month }\end{array}$ & $\begin{array}{l}\text { Surgical resection and } \\
\text { Penicillin }\end{array}$ & No recurrence at 12 months \\
\hline 2 & Single lesion & $\begin{array}{l}\text { Intermittent headache and } \\
\text { dizziness for } \sim 1 \text { month }\end{array}$ & $\begin{array}{l}\text { Surgical resection and } \\
\text { Penicillin }\end{array}$ & No recurrence at 18 months \\
\hline 3 & Single lesion & $\begin{array}{l}\text { Headache and intermittent } \\
\text { vomiting for } \sim 3 \text { months }\end{array}$ & $\begin{array}{l}\text { Surgical resection and } \\
\text { Penicillin }\end{array}$ & No recurrence at 18 months \\
\hline 4 & Multiple lesions (five) & $\begin{array}{l}\text { Headache with decreased muscle } \\
\text { strength for } \sim 6 \text { months }\end{array}$ & $\begin{array}{l}\text { Surgical resection and } \\
\text { penicillin }\end{array}$ & No recurrence at 6 months \\
\hline 5 & Single lesion & $\begin{array}{l}\text { Sudden headache with limb } \\
\text { weakness }\end{array}$ & $\begin{array}{l}\text { Surgical resection and } \\
\text { Penicillin }\end{array}$ & No recurrence at 12 months \\
\hline 6 & Single lesion & $\begin{array}{l}\text { Headache with vomiting for } \\
\sim 2 \text { months }\end{array}$ & Surgical resection & Death one week after surgery \\
\hline
\end{tabular}
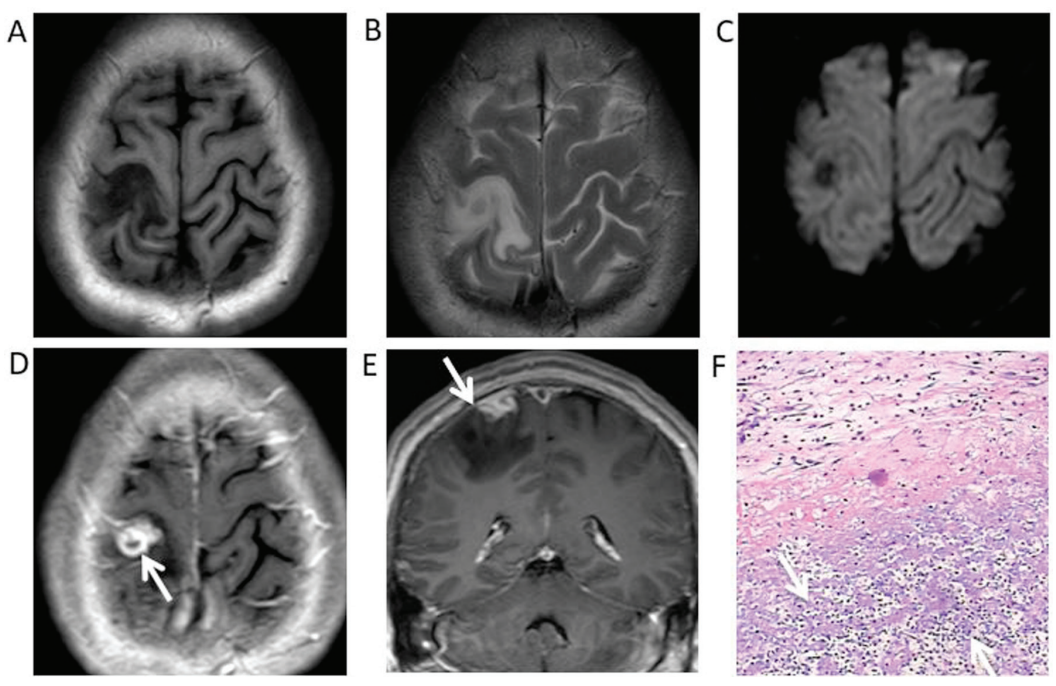

Figure 1. Syphilitic gumma of the right parietal cortex in a 38-year-old male. (A) Magnetic resonance images demonstrated a nodule with perilesional oedema and hypointensity under T1WI, (B) slight hyperintensity in T2WI and (C) hypointensity in diffusion-WI. (D) Postcontrast T1WI revealed a marked ring-like enhancement (white arrow) (E) with a dural tail (white arrow) adjacent gumma. (F) Haematoxylin and eosin staining revealed a small haemorrhage and extensive inflammatory cell infiltration, predominantly of lymphocytes and plasma cells (white arrows), in the gumma (magnification x200). WI, weighted images.

2 of the 6 patients under enhanced T1WI. This was described as 'the dural tail sign' (Fig. 1E).
Whole-body 18F-FDG PET/CT was performed on a single patient (patient 4) before surgery and at 6 months after 

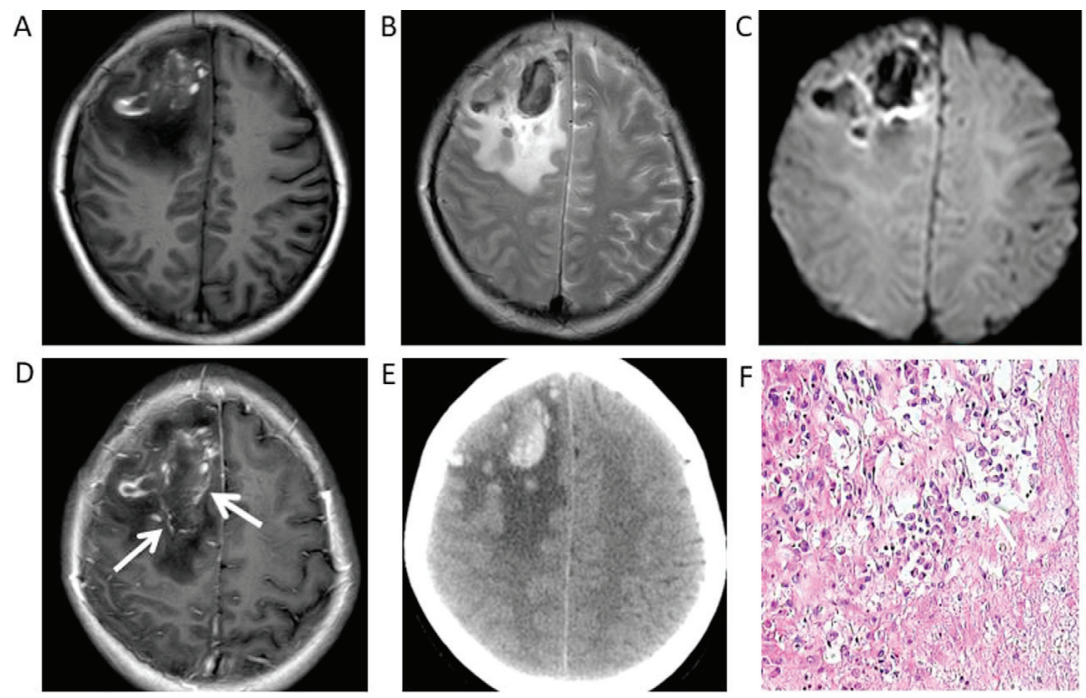

Figure 2. Syphilitic gumma of the right frontal lobe in a 40-year-old female. Magnetic resonance images revealed a heterogeneous signal of the lesion under (A) T1WI, (B) T2WI and (C) diffusion-WI, with surrounding oedema. (D) Postcontrast T1WI revealed the mass with an irregular ring-like enhancement and an adjacent meningeal enhancement. (E) Non-enhanced CT scans demonstrated a haemorrhage within the mass. (F) Haematoxylin and eosin staining revealed extensive inflammatory cell (white arrow) infiltration (magnification x400). WI, weighted images.
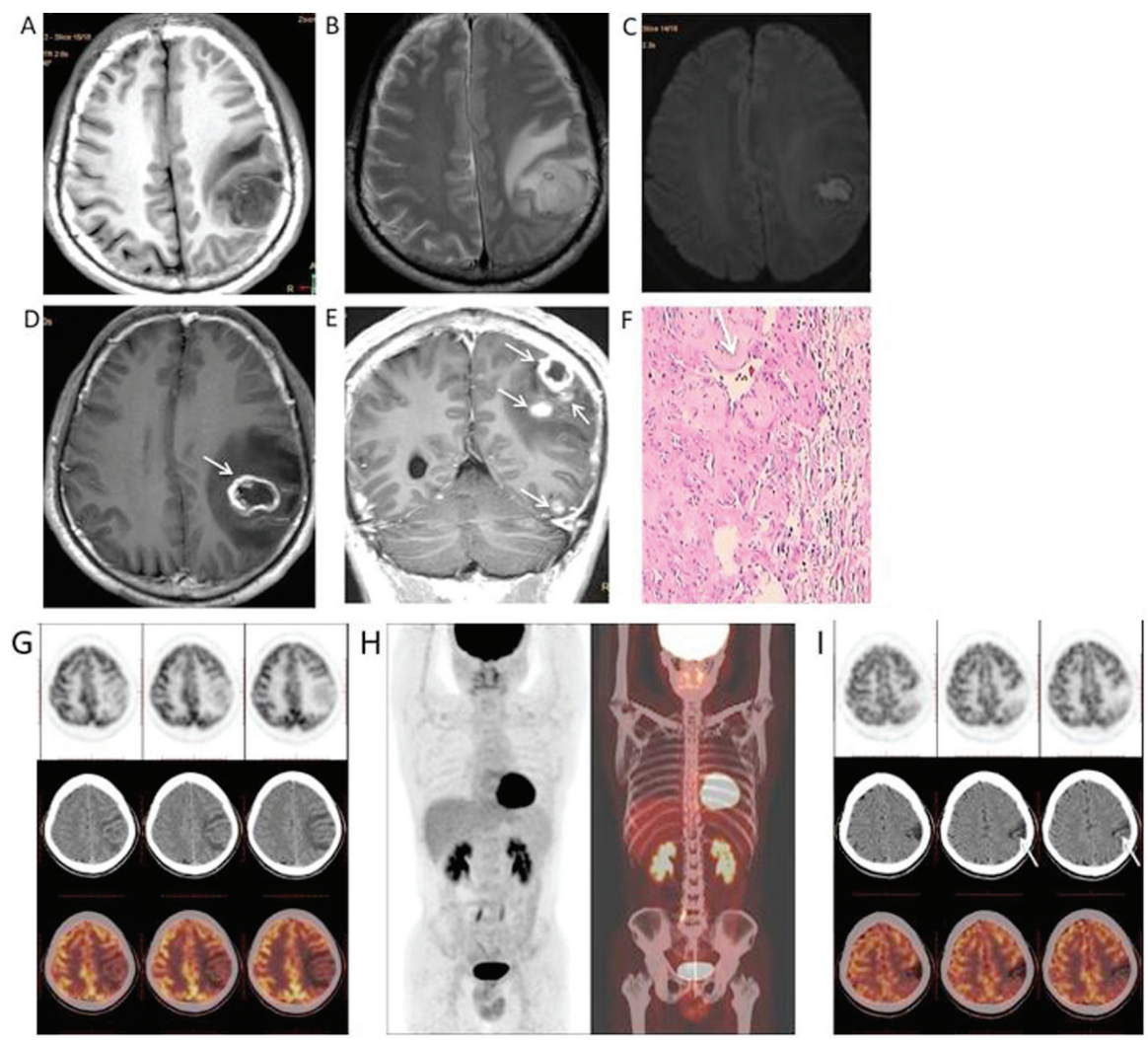

Figure 3. Syphilitic gummata of the left cerebral hemisphere in a 50-year-old male. (A) Magnetic resonance images revealed a round mass with hypointensity under T1WI, (B and C) hyperintensity under T2WI and diffusion-WI, and a marked ring-like (white arrows) (D) or nodular enhancement (white arrows) (E) on gadolinium-enhanced T1WI. (F) Haematoxylin and eosin staining indicated a small haemorrhage, angiosclerosis (white arrow) and inflammatory cell infiltration, mainly consisting of lymphocytes and plasma cells, in the gumma (magnification x200). (G) Pre-operative whole-body 18F-FDG PET/CT indicated the hypometabolic status of gumma and (H) no other primary or metastatic tumors. (I) An 18F-FDG PET/CT follow-up, 6 months after high-dose penicillin treatment, indicates that the lesions tended to calcify post-operatively. WI, weighted images; 18F-FDG PET/CT, 2-deoxy-2-(fluorine-18)fluoro-D-glucose-positron emission tomography/computerized tomography.

surgery. The first 18F-FDG PET/CT examination revealed low uptake and low metabolism in the gumma (Fig. 3G) and 18F-FDG uptake in the gumma was lower than that of normal grey matter (Fig. 3G). No other primary or metastatic tumors were identified in the patient (Fig. 3H). Follow-up 18F-FDG $\mathrm{PET} / \mathrm{CT}$ scans at 6 months after high-dose penicillin treatment revealed that the lesions tended to calcify post-operatively (Fig. 3I). 

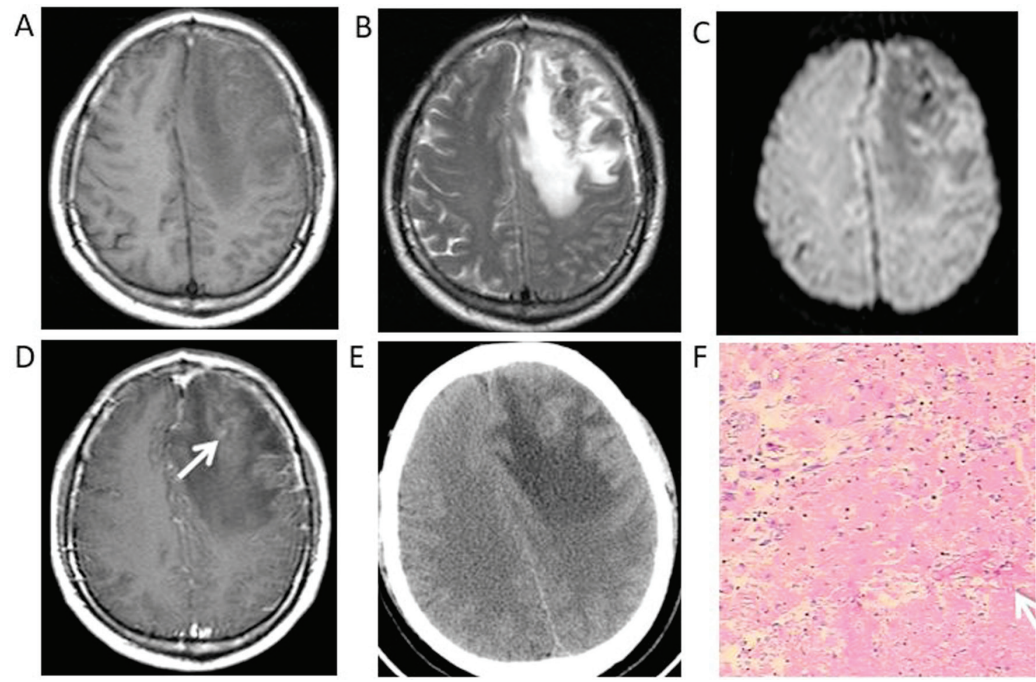

Figure 4. Syphilitic gumma of the left frontal lobe in a 56-year-old male. (A) Magnetic resonance images indicated an irregular lesion with surrounding oedema, hyper/isointensity under T1WI, (B) hypo/isointensity under T2WI, (C) hypointensity under diffusion-WI and (D) a mild strip-like enhancement on gadolinium-enhanced T1WI. (E) A non-enhanced CT scan revealed a slight high-density lesion. (F) Haematoxylin and eosin staining indicated a multifocal haemorrhage (white arrow) and cheese-like necrosis in the pathological tissues, with lymphocyte and plasmocyte infiltration, glial proliferation and intimal hyperplasia around necrotic foci (magnification x200). WI, T1-weighted images.
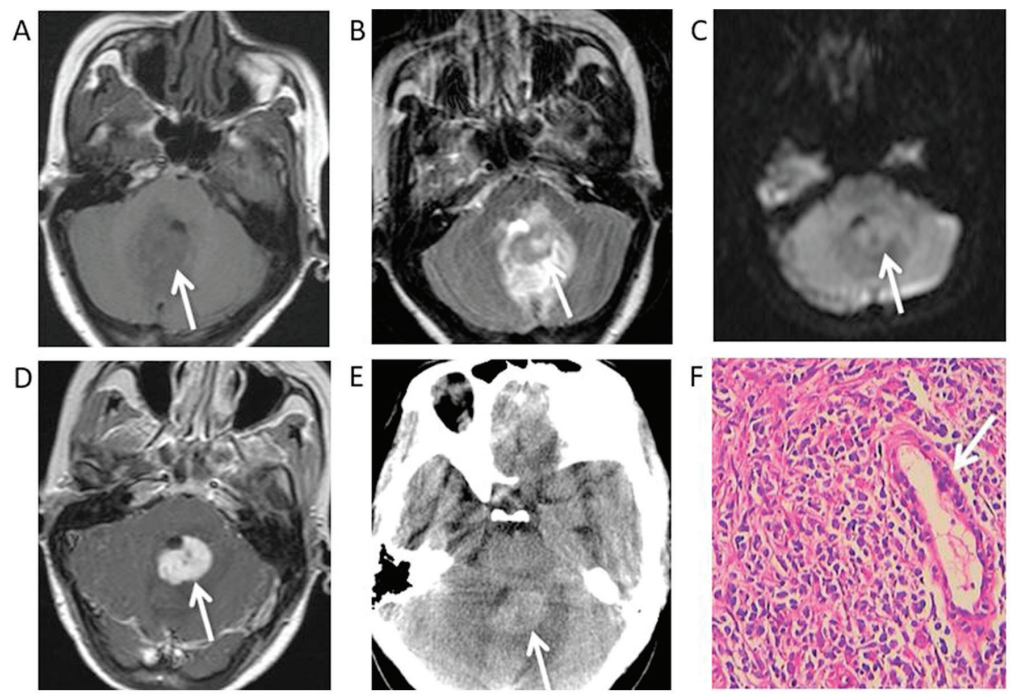

Figure 5. Syphilitic gumma in the fourth ventricle of a 32-year-old female. (A) Magnetic resonance images indicated a round mass, hypointensity under T1WI, (B) hyperintensity under T2WI, (C) slight hyperintensity under diffusion-WI and (D) a marked uniform enhancement on gadolinium-enhanced T1WI. (E) An unenhanced CT scan demonstrated a slight high-density mass. (F) Haematoxylin and eosin staining revealed that many lymphocytes, monocytes and plasma cells had infiltrated the gumma tissue with small vessel hyperplasia (white arrow) and fibrous tissue proliferation (magnification x400). WI, weighted images.

The pathological features. Syphilitic gumma is a classic example of granulomatous inflammation. Microscopic examination showed that each of the resected lesions had multifocal haemorrhage (Fig. 4F) and necrosis with small necrotic vessels (Figs. 1F, 3F). There was also prominent perivascular chronic inflammatory lymphocyte and plasma cell infiltration surrounding areas of necrosis (Figs. 2F, 5F).

\section{Discussion}

Syphilis is a chronic infectious disease caused by Treponema pallidum that is distinguishable among other infectious diseases due to its large variety of clinical manifestations $(2,4)$. According to guidelines from the Centers for
Disease Control and Prevention, syphilis can be divided into the following four phases: Primary, secondary, latent and tertiary (12). Neurosyphilis can occur at each of these stages with various clinical presentations, including cranial nerve dysfunction, meningitis and acute or chronic changes in mental status. The main neuroimaging manifestations include diffuse cerebral atrophy followed by cerebral infarction (13). However, neurosyphilis may also be asymptomatic, which frequently leads to a lack of diagnosis (14). Cerebral syphilitic gumma is a rare manifestation of advanced meningovascular syphilis that has presented following the introduction of penicillin and usually occurs 10-30 years after contracting syphilis (12). However, the progression of syphilis is reported to be faster in patients with HIV compared with those not infected with 
Table III. Neuroimaging findings of cerebral syphilitic gumma.

\begin{tabular}{|c|c|c|c|c|c|c|c|}
\hline Case & T1WI & T2WI & DWI & Enhancement patterns & Dural tail sign & $\mathrm{CT}$ & VE \\
\hline 1 & Hyper/iso intensity & Hypo/iso intensity & Hypo intensity & Mild strip-like & No & High density & III \\
\hline 2 & Hypo intensity & Hyper intensity & Hypo intensity & Marked ring-like & Yes & Low density & I \\
\hline 3 & Hypo intensity & Hyper intensity & Hypo intensity & Marked strip-like & No & Low density & II \\
\hline 4 & Hypo intensity & Hyper intensity & Hyper intensity & Marked ring-like & No & Low density & II \\
\hline 5 & Hyper/hypo intensity & Hypo/iso intensity & Hypo intensity & Marked ring-like & Yes & High density & II \\
\hline 6 & Hypo intensity & Hyper intensity & Iso intensity & Marked homogeneous & No & High density & II \\
\hline
\end{tabular}

T1WI, T1-weighted image; T2WI, T2-weighted image; DWI, diffusion weighted image; VE, vasogenic oedema.

HIV (15). A definitive diagnosis can be difficult as syphilitic gumma can present with a variety of central nervous system manifestations that mimic other diseases, including malignant neoplasms and other inflammatory diseases. In the present study, the clinical manifestations of 6 patients were assessed and were determined to be unspecific. Of the 6 patients, 3 patients experienced headaches and vomiting, 2 patients exhibited headaches and decreased muscle strength, and a single patient suffered recurrent dizziness. None of the patients had typical lesions of stage three syphilis, such as erythema and papules. According to literature and the author's clinical experience, the clinical manifestations of syphilitic gumma are not specific for its diagnosis $(9,11)$. The course, symptoms and signs of the disease were not significantly different from those of other brain lesions, including tumors, tuberculosis and abscesses, except for the history of syphilis. However, in some cases, the history of syphilis provided by patients is not objective or is inaccurate as many patients with syphilis tend not to disclose their disease history. Of the 6 cases assessed in the present study, 4 cases were misdiagnosed as glioma, a single case was misdiagnosed as a metastatic tumor and another case was misdiagnosed as ependymoma. Neuroimaging may provide an insight into the pathology of the diverse clinical manifestations of gumma. MRI is the best choice for detecting central nervous system diseases. Compared with CT, the signal characteristics of MRI more accurately and objectively reflect the pathological features of the lesions being investigated (16-18). However, CT is more sensitive for small calcifications, which is helpful for the differential diagnosis of central nervous system diseases (19).

Fargen et al (20) reported that cerebral syphilitic gummata were more common in men $(64 \%)$ and in those aged between 18-39 years in 156 cases that presented with 185 lesions. Additionally, lesions may be located anywhere, but were most common on the convexities (66\%), as cerebral gumma is thought to arise from a direct extension of syphilitic meningovascular inflammation into the adjacent brain. This inflammation usually entails extension from the pia mater or via small intracerebral vessels that course into the subcortical grey nuclei. In the present study, all lesions were located in a superficial region of the cerebral hemisphere, except in a single case where the lesion was located in the fourth ventricle. The results of the present study are consistent with the findings of this aforementioned study.

MRI generally demonstrated hypointensity under T1WI and hyperintensity under T2WI with adjacent oedema, while CT images revealed low density results. Occasionally, syphilitic gumma was accompanied by haemorrhage, necrosis or calcification, with a heterogeneous signal. In the present study, all the masses performed as ring-like, strip-like or uniform enhancements under gadolinium-enhanced T1WI. The enhancement pattern of the lesion was associated with the disrupted blood-brain barrier in the peripheral blood vessels of inflammatory granulation tissue $(7,8,13)$. In the present study, 2 cases of dural enhancement were identified adjacent to masses, which were deemed 'dural tail sign'. Bourekas et al (21) proposed that gummas are mass lesions of inflammatory granulomatous tissue. It was therefore hypothesized that the dural tail is caused by reactive changes in adjacent connective tissue and by hypervascularity. Additionally, pathological specimens frequently exhibit dural thickening and inflammation adjacent to cerebral gumma (7). The dural tail sign has also been reported in previous studies on syphilitic gumma $(7-9,20,21)$. In a previous study, Tsuboi et al (22) revealed that all the cases studied exhibited marked enhancement with gadolinium administration, with $\sim 35 \%$ of cases presenting with perilesional meningeal enhancement and thickening or a dural tail via MRI. Massive haemorrhage was also accompanied by syphilitic gumma in a female patient. Therefore, the present study speculated that the cause of haemorrhage may be associated with vascular endothelial cell injury and the long-term inflammatory stimulation of the vascular wall, leading to the rupture of small vessels. A single case analyzed in the present study with left hemisphere lesions of different sizes and ring-like enhancement on gadolinium-enhanced T1WI was misdiagnosed as brain metastasis before surgery. The patient was pre-operatively subjected to a whole-body $18 \mathrm{~F}-\mathrm{FDG}$ PET-CT to determine whether there were other primary or metastatic tumors, but none were identified. 18F-FDG PET/CT examination revealed gumma exhibited a lower metabolism and lower uptake of 18F-FDG, and the 18F-FDG uptake by the gumma was lower than that of the surrounding normal grey matter. Follow-up 18F-FDG PET-CT scans 6 months after high-dose penicillin treatment, revealed that the lesions tended to calcify post-operatively. To the best of our knowledge, there are no studies assessing the 18F-FDG PET/CT results of syphilis gumma. Similarly, in one case report of syphilitic gumma, CT perfusion revealed no increase in the cerebral blood volume of the enhancing lesion compared with the ipsilateral normal-appearing white matter (23). The 6th case in the present study that had a mass in the fourth 
ventricle with uniform enhancement on gadolinium-enhanced T1WI was highly suspected to be ependymocytoma based on pre-operative MRI. In the present study, it was difficult to distinguish between the gumma and ependymocytoma, according to the neuroimaging findings. Due to the various forms of intracranial gumma images and a lack of radiologist experience, pre-operative misdiagnosis occurs frequently in clinical practice.

Intracranial syphilitic gumma have extensive differential diagnoses, including glioma, metastasis, malignant meningioma and abscess. Malignant gliomas possess profuse neovascularisation characterized by disorganized, irregular and tortuous vessels with arteriovenous shunting (24). An irregular tumor vascular structure consequently causes abnormal vascular function with increased permeability and perfusion $(24,25)$. Malignant gliomas are characterized by increased perfusion and heterogeneous disruption of the blood-brain barrier (26). The tumor exhibits irregular ring-like or nodular enhancement under gadolinium-enhanced T1WI and its margin presents vasogenic oedema with infiltrative tumor cells along with perivascular spaces (24-26). However, gumma is a chronic perivascular inflammation that is characterized by the proliferation of small vessels and the infiltration of lymphocytes and plasmocytes around regions of necrosis. Patients with metastases usually exhibit a history of primary malignant tumors and mostly occur in grey and white matter junctions. Malignant meningioma presents features of an aggressive disease, with no demarcation between the tumor and brain parenchyma, and invasion of surrounding structures, including the skull, scalp and venous sinus. They characteristically invade the brain in a mushroom shape from their dural attachment $(27,28)$. However, syphilitic gumma encroaches on and is closely associated with the meninges. The edge of the lesion often intersects with the surrounding meninges at an obtuse angle (2). The pus of most abscesses show as hyperintense under DWI, hyperintense under T2WI and as a peripheral hypointense rim and iso- to hypointense under T1WI, which are characteristics of integument-term brain abscesses (29).

Functional MRI techniques, including magnetic resonance spectroscopy (MRS), perfusion weighted imaging (PWI) and DWI, are helpful for the diagnosis and differential diagnosis of tumors and non-neoplastic lesions. The proton-MRS technique produces an increase in the choline $(\mathrm{Cho}) /$ creatine $(\mathrm{Cr})$ ratio and a reduction in the $\mathrm{N}$-acetylaspartate (NAA)/Cr ratio in brain tumors (30). In a previous study published by Ventura et al (31), a mild increase in the Cho/NAA ratio in syphilitic gumma was identified. Furthermore, the brain PWI reflected the degree of tumor vascular proliferation and vascular permeability, but PWI is not directly associated with damage of the blood-brain barrier $(32,33)$. The perfusion of high-grade glioma was significantly increased, while infectious lesions, such as brain abscess, were significantly reduced $(34,35)$. To the best of our knowledge, there are no studies on the magnetic resonance perfusion imaging of intracranial syphilis gumma. However, it is speculated that functional MRI may be helpful in the diagnosis and differential diagnosis of intracranial syphilitic gumma.

In conclusion, two important points are suggested regarding the neuroimaging findings of intracranial syphilitic gumma. First, syphilitic gumma predominantly appeared in the superficial part of the cerebral hemisphere, which mostly involved the grey matter. Second, meningeal thickening and enhancement adjacent to syphilitic gumma could be of great significance. These two points, combined with advanced neuroimaging techniques and laboratory examinations could aid accurate pre-operative diagnoses.

\section{Acknowledgements}

Not applicable.

\section{Funding}

No funding was received.

\section{Availability of data and materials}

The datasets used and/or analyzed during the current study are available from the corresponding author on reasonable request.

\section{Authors' contributions}

CL and GC collected and analysed the clinical data and prepared the manuscript; LL, SW and GT collected the clinical data. The final version of the manuscript has been read and approved by all authors and each author believes that the manuscript represents honest work.

\section{Ethical approval and consent to participate}

The present study was approved by the Ethics Committee of Affiliated Hospital of Southwest Medical University.

\section{Patient consent for publication}

Patients provided written informed consent for publication.

\section{Competing interests}

The authors declare that they have no competing interests.

\section{References}

1. World Health Organization (WHO): WHO guidelines for the treatment of Treponema pallidum (syphilis). WHO, Geneva, 2016. www.who.int/reproductivehealth/publications/rtis/syphilis-treatment-guidelines/en/.

2. Grillova L, Jolley K, Smajs D and Picardeau M: A public database for the new MLST scheme for Treponema pallidum subsp. pallidum: Surveillance and epidemiology of the causative agent of syphilis. Peer J 6: e6182, 2019.

3. Newman L, Rowley J, Vander Hoorn S, Wijesooriya NS, Unemo M, Low N, Stevens G, Gottlieb S, Kiarie J and Temmerman M: Global estimates of the prevalence and incidence of four curable sexually transmitted infections in 2012 based on systematic review and global reporting. PLoS One 10: $\mathrm{e} 0143304,2015$.

4. Gong XD, Yue XL, Teng F, Jiang N and Men PX: Syphilis in China from 2000 to 2013: Epidemiological trends and characteristics. Chin J Dematol 47: 310-315, 2014.

5. Clark EG and Danbolt N: The Oslo study of the natural course of untreated syphilis: An epidemiologic investigation based on re-study of the Boeck-Bruusgaard material. Med Clin North Am 48: 613-623, 1964. 
6. Roeske LC and Kennedy PR: Images in clinical medicine. Syphilitic gummas in a patient with human immunodeficiency virus infection. N Engl J Med 10: 1123, 1996.

7. Shao XF, Qiang D, Liu YH, Yuan Q, Tao J and Ji BH: Diagnosis and treatment of cerebral syphilitic gumma: A report of three cases. Front Neurosci 27: 100, 2018

8. Xia DY, Zhu MF, Liu CG, Dai Y, Li ZB, Jiang XC and Xu SS Cerebral syphilitic gumma misdiagnosed as a malignant brain tumor. J Craniofac Surg 28: e170-e172, 2017.

9. Baek HJ and Kim WJ: Cerebral gumma mimicking a brain tumor in a human immunodeficiency virus-negative patient: A case report. J Korean Soc Radiol 69: 181-185, 2013.

10. Kikuchi Y, Hiwatashi A, Togao O, Yamashita K, Momosaka D and Honda $\mathrm{H}$ : Cerebral syphilitic gumma mimicking glioma: Utility of CT perfusion. Diagn Interv Imaging 99: 755-757, 2018

11. Agrons GA, Han SS, Husson MA and Simeone F: MR imaging of cerebral gumma. Am J Neuroradiol 12: 80-81, 1991.

12. Fan SR and Liang LF: CDC 2015 guideline for the diagnosis and treatment of syphilis. Chin Gen Pract 18: 3260-3264, 2015.

13. Nagappa M, Sinha S, Taly AB, Rao SL, Nagarathna S, Bindu PS Bharath RD and Murthy P: Neurosyphilis: MRI features and their phenotypic correlation in a cohort of 35 patients from a tertiary care university hospital. Neuroradiology 55: 379-388, 2013.

14. Wang Z, Liu L, Shen YZ, Zhang RF, Qi TK, Tang Y, Song W, Chen $\mathrm{J}$ and $\mathrm{Lu} \mathrm{H}$ : The clinical and laboratory features of neurosyphilis in HIV-infected patients: A retrospective study in 92 patients. Medicine 97: e0078, 2018.

15. Johns DR, Tierney M and Felsenstein D: Alteration in the natura history of neurosyphilis by concurrent infection with the human immunodeficiency virus. N Engl J Med 316: 1569-1572, 1987.

16. Scatliff JH: MRI of the central nervous system. Investigative Radiol 26: 622-623, 1991.

17. Mori K: Introduction. In: Mori K, (eds) MRI of the Central Nervous System. Springer, Tokyo, 1991.

18. Kimura M and da Cruz LCH Jr: Multiparametric MR imaging in the assessment of brain tumors. Magn Reson Imaging Clin $\mathrm{N}$ Am 24: 87-122, 2016.

19. Laughlin S and Montanera W: Central nervous system imaging When is CT more appropriate than MRI? Postgrad Med 104 73-76, 81-84, 87-88, 1998.

20. Fargen KM, Alvernia JE, Lin CS and Melgar M: Cerebral syphilitic gummata: A case presentation and analysis of 156 reported cases. Neurosurgery 64: 568-576, 2009.

21. Bourekas EC, Wildenhain P, Lewin JS, Tarr RW, Dastur KJ, Raji MR and Lanzieri CF: The dural tail sign revisited. AJNR Am J Neuroradiol 16: 1514-1546, 1995.

22. Tsuboi M, Nishijima T, Teruya K, Kikuchi Y, Gatanaga H and Oka S: Cerebral syphilitic gumma within 5 months of syphilis in HIV-infected patient. Emerg Infect Dis 22: 1846-1848, 2016.
23. Kikuchi Y, Hiwatashi A, Togao O, Yamashita K, Momosaka D and Honda $\mathrm{H}$ : Cerebral syphilitic gumma mimicking glioma: Utility of CT perfusion. Diagn Interv Imaging 99: 755-757, 2018

24. Soda Y, Myskiw C, Rommel A and Verma IM: Mechanisms of neovascularization and resistance to anti-angiogenic therapies in glioblastoma multiforme. J Mol Med (Berl) 91: 439-448, 2013.

25. Jain RK, di Tomaso E, Duda DG, Loeffler JS, Sorensen AG and Batchelor TT: Angiogenesis in brain tumours. Nat Rev Neurosci 8: 610-622, 2007.

26. Zhang J, Liu H, Tong H, Wang S, Yang Y, Liu G and Zhang W: Clinical applications of contrast-enhanced perfusion MRI techniques in gliomas: Recent advances and current challenges. Contrast Media Mol Imaging 2017: 7064120, 2017.

27. O'Leary S, Adams WM, Parrish RW and Mukonoweshuro W: Atypical imaging appearances of intracranial meningiomas. Clin Radiol 62: 10-17, 2007.

28. Lyndon D, Lansley JA, Evanson J and Krishnan AS: Dural masses: Meningiomas and their mimics. Insights Imaging 10: 11, 2019.

29. Gupta RK, Hasan KM, Mishra AM, Jha D, Husain M, Prasad KN and Narayana PA: High fractional anisotropy in brain abscesses versus other cystic intracranial lesions. Am J Neuroradiol 26: 1107-1114, 2005.

30. Son HS, Kim EN, Kim SH, Yoo YR, Jung YA, Jung SG, Hong YG, Lee YS and Choi BY: Evaluation of Glioma with Thallium-201 Brain SPECT: The correlation with $1 \mathrm{H}$ MR spectroscopy and pathology. Korean J Nucl Med 34: 465-477, 2000.

31. Ventura N, Cannelas R, Bizzo B and Gasparetto EL: Intracranial syphilitic gumma mimicking a brain stem glioma. AJNR Am J Neuroradiol 33: E110-E111, 2012.

32. Maia AC Jr, Malheiros SM, da Rocha AJ, da Silva CJ, Gabbai AA, Ferraz FA and Stávale JN: MR cerebral blood volume maps correlated with vascular endothelial growth factor expression and tumor grade in nonenhancing gliomas. AJNR Am J Neuroradiol 26: 777-783, 2005.

33. Fu L and Li K: Principles \& clinical application of brain PWI. Chin Comput Med Imag 19: 180-183, 2013 (In Chinese).

34. Britt RH, Enzmann DR and Yeager AS: Neuropathological and computerized tomographic findings in experimental brain abscess. J Neurosurg 55: 590-603, 1981.

35. Hakyemez B, Erdogan C, Ercan I, Ergin N, Uysal S and Atahan S: High-grade and low-grade gliomas: Differentiation by using perfusion MR imaging. Clin Radiol 60: 493-502, 2005.

(i) $\odot$ This work is licensed under a Creative Commons Attribution-NonCommercial-NoDerivatives 4.0 International (CC BY-NC-ND 4.0) License. 